\begin{abstract}
ANL-01/15
ARGONNE NATIONAL LABORATORY

9700 South Cass Avenue

Argonne, Illinois 60439-4801
\end{abstract}

\title{
ALTERNATIVE IMPLEMENTATIONS \\ OF THE MONTE CARLO POWER METHOD
}

by

R. N. Blomquist and E. M. Gelbard

Reactor Analysis and Engineering Division

December, 2001 


\section{TABLE OF CONTENTS}

$\underline{\text { Page }}$

ABSTRACT iv

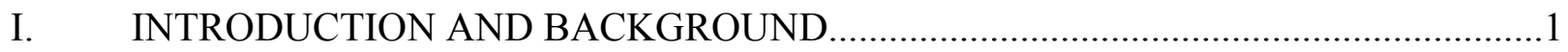

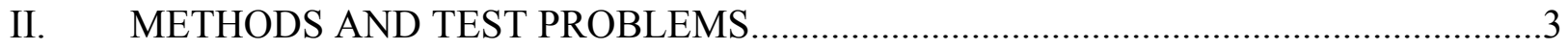

A. UNIFORM THREE-REGION ONE-GROUP SLAB CELLS ..............................

B. NON-UNIFORM THREE-REGION ONE-GROUP SLAB CELLS.....................5

C. JAERI TWO-CORE CRITICALITY SAFETY PROBLEM.................................5

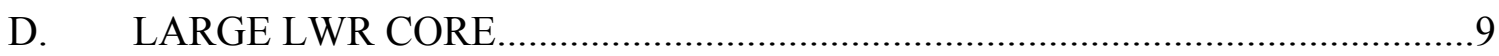

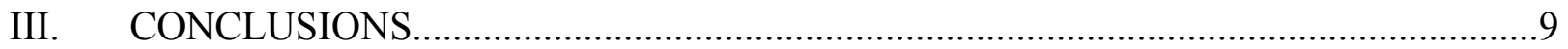

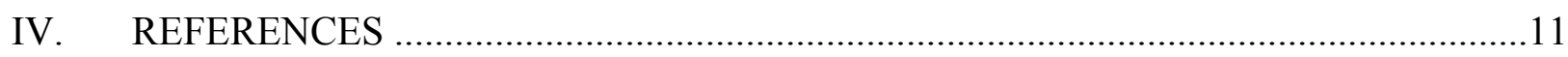

APPENDIX A: THEORY OF VARIANCE DUE TO RANDOM SOURCE-RESAMPLING

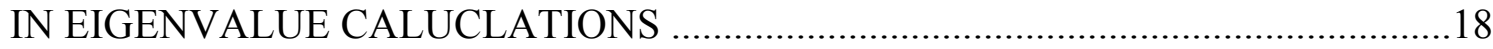

APPENDIX B: THEORY OF VARIANCE INDUCED THROUGH USE OF COLLISION SITES AS POTENTIAL FISSION SITES .............................................22

APPENDIX C: THEORY OF VARIANCES IN AVERAGES OVER GENERATIONS.........27

APPENDIX D: THEORY OF COUPLING AND SOURCE DISTRIBUTION

FLUCTUATIONS 


\section{LIST OF FIGURES}

Page

1. Schematic View of JAERI Coupled Slabs System .....................................................12

2. JAERI Slabs: Ratio of Starting Neutrons (Slab 1 / Slab 2) in Each Generation ...............12

3. JAERI Slabs: Maximum Fission Ratios for 20 Replicas .................................................13

\section{LIST OF TABLES}

I. Absorption and $\mathrm{k}_{\mathrm{eff}}$ Variances for Uniform Three-Region One-Group Slabs ..........14

II. Absorption Rate Variances, $\mathrm{k}_{\mathrm{eff}}$ and Variances in Non-Homgeneous Slabs...............14

III. JAERI Problem, One-Group Parameters …………....................................................

IV. Computational Characteristics: One-Group JAERI Problem ……...................................15

V. JAERI Problem: One-Group Monte Carlo Results .....................................................15

VI. VIM Continuous-Energy Results, Collision Site Technique ............................................15

VII. VIM Continuous-Energy Results, Absorption Site Technique …………......................15

VIII. Homogeneous LWR Atom Densities ……………................................................

IX-A. Homogeneous LWR Results: Continuous Energy, Absorption-Sites

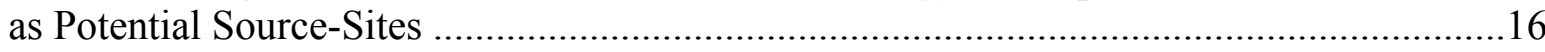

IX-B. Homogeneous LWR Results: Continuous Energy, Collision-Sites as Potential Source-Sites ..............................................................................................16

X. Heterogeneous LWR Atom Densities …………................................................17

XI-A. Heterogeneous LWR Results: Continuous Energy, Absorption Sites as Potential Source Sites

XI-B. Heterogeneous LWR Results: Continuous Energy, Collision Sites as Potential Source Sites 


\title{
Alternative Implementations of the Monte Carlo Power Method
}

\author{
by
}

\author{
R. N. Blomquist and E. M. Gelbard
}

\begin{abstract}
We compare nominal efficiencies, i.e. variances in power shapes for equal running time, of different versions of the Monte Carlo eigenvalue computation, as applied to criticality safety analysis calculations. The two main methods considered here are "conventional" Monte Carlo and the superhistory method, and both are used in criticality safety codes. Within each of these major methods, different variants are available for the main steps of the basic Monte Carlo algorithm. Thus, for example, different treatments of the fission process may vary in the extent to which they follow, in analog fashion, the details of real-world fission, or may vary in details of the methods by which they choose next-generation source sites. In general the same options are available in both the superhistory method and conventional Monte Carlo, but there seems not to have been much examination of the special properties of the two major methods and their minor variants. We find, first, that the superhistory method is just as efficient as conventional Monte Carlo and, secondly, that use of different variants of the basic algorithms may, in special cases, have a surprisingly large effect on Monte Carlo computational efficiency.
\end{abstract}




\section{INTRODUCTION AND BACKGROUND}

The superhistory method was introduced in 1986 in a key paper by Brissenden and Garlick (Ref. 1). This method is designed to reduce substantially the biases induced, both in eigenvalues and flux shapes, by conventional Monte Carlo, the most commonly used criticality safety analysis method. In the Monte Carlo ("MC") power method, as in the deterministic method, the eigenvalue computation is carried out iteratively, in iterations called here "generations". The number of neutrons, $N_{\mathrm{s}}$, present at the start of each generation is usually fixed, in standard MC, via input, and positions of the first $N_{\mathrm{s}}$ starters are usually selected stochastically from a guessed initial distribution. Here we will consider standard MC only in its simplest form, in which each starter is followed until it is absorbed or escapes. When all neutrons in a generation are gone, $N_{\mathrm{s}}$ new starting sites are chosen, by one of several alternative processes, from among real or "potential" fission sites generated in the just-completed generation. These new starters, in their turn, are then followed for another generation, etc., for a user-specified number of generations.

Within each generation, the neutrons may be tracked by any valid process, i.e., by any process such that the expected values of track lengths and reaction rates correspond to correct physical values, given the geometry, physics parameters, and starting locations. There is, however, no real physical process that is modeled by the renormalization of the fission source after every generation, i.e. by the completely artificial readjustment of the number of neutrons participating in the chain reaction. It turns out (Refs. 1,2) that, because of statistical fluctuations in the $\mathrm{MC}$ eigenvalue, this normalization is not an unbiased simulation of normalization in deterministic power iteration. For this reason all MC estimates are biased from the first reselection of $\mathrm{N}_{\mathrm{s}}$ new starting sites, no matter which of the usual alternatives is used to execute this step. This means that beyond this point the expected values will not agree with the true solution of the transport equation for the given cross sections and geometry. There are ways to estimate the resulting eigenvalue bias, and reasons to believe that this bias is usually inconsequential (Refs. 1,2), but we have no good estimate of or bound on biases in flux shapes. For this reason it seems advisable to reduce the biases as much as possible.

The superhistory method does just this by decreasing the frequency of sourcerenormalization. Each starter is now followed for a specified number of generations, $N_{\mathrm{g}}$, and the resulting extended history is now called a "superhistory". More specifically: 1) the starter is followed until it is absorbed or escapes; 2) when absorbed, it may excite a fission producing, on average, the proper number of offspring; and 3) all these are followed for a second generation until they too are absorbed or escape, and so on for $N_{\mathrm{g}}$ generations. Here the set of $N_{\mathrm{g}}$ generations is called a "supergeneration". Each supergeneration provides an eigenvalue estimate equal to the ratio of the net weight of all potential fission sites produced in that supergeneration, to the net weight of all source neutrons in all generations of that supergeneration. Estimates of reaction rates are defined similarly. At the end of each supergeneration $N_{\mathrm{s}}$ new starting locations are chosen from potential starting sites, now produced in the last generation of the preceding supergeneration. It will be seen that conventional MC is just the superhistory method with $N_{\mathrm{g}}=$ 1 . 
In conventional $\mathrm{MC}$ the fluctuations in computed fission distributions are in part counteracted, in the course of each generation, by restoration of the weight of starters to the specified input value. In contrast in the superhistory method this renormalization of the fission source occurs much less frequently, allowing some additional accumulation of source fluctuations. It is one of our goals to determine to what extent effects of this noise accumulation are eliminated by the normalization at the end of the supergeneration.

Above we have described the superhistory method in the form that seems to be recommended in Ref. 1, and described by Nigel Smith. ${ }^{a}$ Absorption sites are potential fission sites, with the fission probability at each site equal to $\Sigma_{\mathrm{f}} / \Sigma_{\mathrm{a}}$, and the average number of offspring from each fission equal to $\bar{v} / k$. Here, $\bar{v}$ is the local average number of neutrons per fission, $k$ is a number close to the system eigenvalue, and we have excluded calculations in which variance reduction methods are applied, so that neutron weights are all unity. The factor $1 / k$ is introduced so that the net number of offspring will be roughly equal to the number of starters, $\mathrm{N}_{\mathrm{s}}$, required in the next generation.

In the variants just described, fission is simulated by a more-or-less analog procedure. Later we refer to this treatment of fission as "Variant A", where "A" signifies "Analog". It is essentially this variant which is used in one of the two versions of the superhistory code MONK (Ref. 3), and in the conventional Russian MC code, MMKFK. ${ }^{\mathrm{b}}$ Probably it is more common, in other MC codes, to use a "less analog" fission simulation. For example one might take each absorption site, as before, as a potential fission site, but now assign to each such site the weight $W_{\mathrm{s}}=\overline{\mathrm{v}} \Sigma_{\mathrm{f}} /\left(\mathrm{k} \Sigma_{\mathrm{a}}\right)$. Fission can now be assumed to occur at each site where this weight is non-zero, with the site weight being the average total weight of neutrons produced by this absorption. In still another fission simulation it is the collision sites which serve as potential new source sites. In this case the site weights will be equal to $W_{\mathrm{s}}=\bar{v} \Sigma_{\mathrm{f}} /\left(\mathrm{k} \Sigma_{\mathrm{t}}\right)$, and it is these weights which then determine the numbers of offspring. In both the collision and absorption simulations, the site weight determines the number of next-generation neutrons emerging from the event. For example, if $W_{\mathrm{s}}=2.5$, either two or three cloned sites will be banked, each with weight 1.0, depending on the roulette of the 0.5 fraction of the site weight. Both of these techniques will be called "Variant E", where "E" is an abbreviation for "expected values". In one form or another, these techniques are the most commonly used. When we refer to Variant E, it will be necessary to say precisely how the potential source sites are defined.

Another set of options is available for resetting the number of starters to $N_{\mathrm{s}}$ at the beginning of each supergeneration. One of these options, which we call "random sourceresampling", will be used in some of our tests though it is rarely seen in practice. We consider this option for various reasons. First, it seems to be indicated in Ref. 1 as an option in the superhistory method. Secondly, it is a very simple way to choose $N_{2}$ starting sites from $N_{1}$ available fission sites and, in addition, is a technique sometimes cited in introductory presentations of MC. By "random source-resampling" we mean here the process applied to a bank containing sites with uniform weights, specified by the following Fortran:

\footnotetext{
${ }^{\text {a }}$ Private communication, Nigel Smith, Serco Assurance (Winfrith), 2001.

${ }^{\mathrm{b}}$ Private communication, Lev Maiorov, Kurchatov Institute, 2001.
} 


$$
\begin{aligned}
& \text { do Neut }=1, \mathrm{~N}_{2} \\
& \text { ccc }=\operatorname{ranf}() \\
& \text { isite }=\operatorname{aint}\left(\operatorname{ccc}^{*} \mathrm{~N}_{1}\right)+1 \\
& X S I n(\text { Neut })=\mathrm{Xf}(\text { isite }) \\
& \text { YSIn(Neut) }=\mathrm{Yf}(\text { isite }) \\
& Z S I n(\text { Neut })=\mathrm{Zf}(\text { isite }) \\
& \text { Enddo }
\end{aligned}
$$

Here $\operatorname{ranf}()$ represents a pseudorandom number drawn from some typical sequence, and the $N_{2}$ XSIn's are the X-coordinates of the required next-generation starters. Clearly if the original sites, (Xf, Yf, Zf), constitute a set of $N_{1}$ points drawn from a given spatial source distribution, then the final (XSIn, YSIn, ZSIn) coordinates constitute a set of $N_{2}$ points drawn from the same distribution. Although the wording in Ref. 1 is not completely clear, the authors seem to imply that they consider this resampling process to be a significant source of noise, and that it may be an advantage of the superhistory method that it reduces the frequency of random sourceresampling; but there are well-known schemes which can replace random source-resampling and are much less noisy. These other techniques can be used in either the conventional or superhistory method, eliminating this particular advantage of the superhistory method.

Finally, there is an option in the formulation of the superhistory method that seems worth mentioning here, though it will not be discussed below. This is a programming option that is very much worth considering. It is possible to arrange a superhistory computation in such a way that it looks very much like conventional MC. That is, the calculation may be run generation by generation, like a conventional $\mathrm{MC}$, but with population control only every $N_{\mathrm{g}}$ generations. One would then have to take account of the fact that the number of starters per generation is variable; but even in conventional MC the number of starters may not be completely constant, varying slightly from one generation to another. This is true, for example, in MCNP (Ref. 4), where, however, the net weight of starters is held constant, and in many of the test computations we will discuss. The advantage of such an approach is that a conventional MC code can be converted into a superhistory code fairly easily. On the other hand one can also arrange the computation so that a superhistory runs to completion before the next superhistory starts. This approach may be advantageous if the MC computation is to be parallelized. In that case the data acquired on different processors must be gathered periodically, and this gathering is usually an expensive operation. In a superhistory computation with $N_{\mathrm{g}}=10$ (the default value in MONK) a superhistory runs much longer than a single history so that the gathering of data, one might expect, need be done relatively infrequently.

\section{METHODS AND TEST PROBLEMS}

The test problem configurations used in this investigation fit into four groups. The first set consists of three identical one-dimensional one-group homogeneous slabs. Each case in this set was run with a different method/variant combination, but all were run with random sourcerenormalization, a process found to be disadvantageous, as predicted in Ref. 1. The second set also consists of three one-dimensional one-group slab cells, now not homogeneous. Random source-resampling is replaced by an alternative that almost sets the number of starters to the 
desired value. Here, this renormalization technique seems to introduce no significant extra noise. Problems in both these sets were run with absorption sites taken as potential next-generation source sites, using Variants $\mathrm{E}$ and $\mathrm{A}$. The third group is a symmetric JAERI two-core problem (Ref. 5) run on VIM (Ref. 6) versions using absorption-based and collision-based source sites. The anomalies in Ref. 5 were reproduced, but results with collision sites as potential source sites were much worse than those using absorption sites. The fourth group is a set of VIM computations modeling a hypothetical large three-dimensional thermal reactor, both in a homogenized form and with a plate structure. This set was an attempt to assess how important homogeneity is as a factor in the performance of different site generation schemes.

\section{A. Uniform Three-Region One-Group Slab Cells}

This problem configuration is a uniform, one-dimensional reflected slab, $15.5 \mathrm{~cm}$ thick, divided into 3 equally thick edit zones. In each we estimate the absorption rate and its variance. In addition we compute the eigenvalue and its variance, using collision estimators throughout. Table I lists the problem parameters, the variance, $\sigma_{\mathrm{k}}{ }^{2}$, in eigenvalues, and the variance, $\sigma_{\mathrm{a}}{ }^{2}$, of absorption rates averaged over edit zones. In the methods of Table I, fission is allowed to occur only at absorption sites.

In the first case with $\bar{v}=1$ and one generation per supergeneration, the superhistory method is equivalent to "conventional" MC. Row 2 displays results for the same slab with $\bar{v}$ raised to 3 and $\Sigma_{\mathrm{f}}$ correspondingly lowered. The row 3 method is identical to row 1 , but with 10 generations per superhistory. All cases correspond to the same just-critical cell, but with factorization of $\bar{v} \Sigma_{\mathrm{f}}$ as an independent parameter. Variances were computed among 1000 replicas, i.e., identical but statistically independent calculations. Closely similar results were obtained for $\bar{v}=2.5$.

It should be understood that the cross sections and dimensions in this set of tests were inherited from earlier studies, and have no special significance. On the other hand it is our hope that, considering the whole range of our test problems, it will be possible to come to reasonably well-founded conclusions.

We see that Variant A flux shapes (Table I, row 2) are noisier than the shapes generated by conventional $\mathrm{MC}$, probably because more than one neutron will emerge from each fission site. Thus the number of fission sites will be smaller in Variant A than in conventional MC, so that less information will reside in the fission source.

In the third computation of Table I, random selection of source sites occurs every tenth generation, while in the first MC it occurs after each generation. Otherwise the computations are identical. Apparently the additional random source resampling produces the observed increase in variance.

One can, however, easily eliminate this source-resampling in all methods discussed above. In conventional MC, for example, we define $w(i)$, the site weight for the $i^{\prime}$ th site, as:

$$
w(i)=\bar{v}(i) \Sigma_{\mathrm{f}}(i) /\left(k \Sigma_{\mathrm{a}}(i)\right)
$$


Let $W_{\mathrm{t}}$ be the sum of all the $w(i)$. Also, let $w^{\prime}(i)=w(i) N_{s} / W_{t}$, where $N_{\mathrm{s}}$ is the number of starters desired at the beginning of each generation or supergeneration. To start a generation or supergeneration, now use $w^{\prime}$ instead of $w$ to determine the mean number of offspring at site $i . N_{\mathrm{s}}$ will now be the expected number of starters. Conventional and superhistory variant E MC calculations using a test code have been modified in this way for the problems studied below.

It might be argued that, by not firmly fixing the number of starters in our "conventional" MC computations, we have artificially suppressed part of the difference between conventional and superhistory variances. Later, however, in discussing the JAERI two-core problem, we compare results obtained via the above techniques with corresponding results from VIM, a code in which the number of starters is constant. Differences between variances in the two MC computations are small, and probably not significant. It must be conceded, however, that properties of this population control mechanism (preceding paragraph) require further examination, and more intercomparison with other methods. Among the most plausible alternatives is a method used by Forrest Brown. ${ }^{\mathrm{c}}$ If the number of potential sites, $N_{\mathrm{p}}$, is greater than the required number of starters, $N_{\mathrm{s}}$, Brown's approach is to randomly select $N_{\mathrm{p}}-N_{\mathrm{s}}$ sites for elimination. If, on the other hand, $N_{\mathrm{s}}$ is greater than $N_{\mathrm{p}}$, then the required additional sites are selected, without replacement, from the available potential sites. Here it is assumed that $N_{\mathrm{s}}$ is not greater than $2 N_{\mathrm{p}}$. Like most others, Brown's method starts from fission sites whose weights have been adjusted to unity by some prior statistical process. A rather different method, called "the comb" was brought to our attention by T. Booth (Ref. 7). The comb starts from any number of weighted fission sites and yields a specified number of unit weight source sites. So far as we know the statistical properties of this method, as applied to reactor eigenvalues, have never been examined.

\section{B. Non-Uniform Three-Region One-Group Slab Cells}

This problem set is physically the same as the previous set, except that in both end slabs, $\Sigma_{\mathrm{f}}=.1 / \bar{v}, .05 / \bar{v}$ and 0 , in problems $1-3$, respectively. The computational methods and variants for this set and the corresponding results are shown in Table II. Here, "Conventional" means one generation per supergeneration, 130 supergenerations run, of which the first 30 are skipped. Variant A means 10 generations per supergeneration, 13 supergenerations run, of which 3 are skipped. Variant E used the same generation parameters as did the Variant A calculations.

Again one sees, as in Table I, that Variant A flux shapes are significantly more noisy than those computed by conventional MC. On the other hand conventional MC and the Variant $\mathrm{E}$ version of the superhistory method are now equally efficient for this expanded set of problems. The disadvantages of random source resampling, on the one hand, and the quasi-analog fission treatment on the other, have both been eliminated.

\section{JAERI Two-Core Criticality Safety Problem}

The test problems in the first two sets were very simple artificial problems convenient for this study. They were particularly useful because they could be run very quickly in many replicas, so that true variances could be computed with great accuracy. Further, they could be run

\footnotetext{
${ }^{\mathrm{c}}$ Private communication, Forrest Brown, Los Alamos National Laboratory, 2001.
} 
on simplified, specially written test codes embodying the superhistory method, an approach that was necessary since ANL does not now have a full-scale implementation of this method.

On the other hand, these test problems do cover only a small range of not very typical configurations. The problem examined here, although still not typical, is very different from the earlier test problems, and was one of the cases encountered in practice by Yamamoto, et al (Ref. 5 ). The problem geometry is depicted in Figure 1 (in our case with $T_{1}=35$ and $T_{\mathrm{c}}=30$ ), and it seems that the simplest way to describe the problem is to quote Ref. 5, as we do below.

"In this paper, two slab fuel solutions are taken as examples of calculation. Each slab is $69 \mathrm{~cm}$ wide and $50 \mathrm{~cm}$ high, and is made of uranyl nitrate aqueous solution. The concentration of uranium is approximately $310 \mathrm{~g} / \mathrm{l}$ and the enrichment is $10 \mathrm{wt} \%$. As shown in Figure 1, two slabs are separated by an ordinary concrete slab of $69 \mathrm{~cm}$ wide and $50 \mathrm{~cm}$ high. The thickness of the unit 2 in Figure 1 is fixed at $35 \mathrm{~cm}$ and the thickness of unit $1, T_{1}$, and the concrete slab, $T_{C}$, are variable. A criticality calculation using MCNP 4B was carried out for a symmetric configuration. The thickness of unit 1 and concrete slab was $35 \mathrm{~cm}$, and $30 \mathrm{~cm}$ respectively. The calculations were performed using active 500 generations of 2,000 neutrons each, after skipping 50 generations. All neutrons in the first generation started with a flat distribution over the fissile material in the system."

Yamamoto and his coworkers found, in repeated calculations, that although in the symmetric case the MC flux guess was symmetric, and although the number of starters per generation was pretty large, still very large asymmetries developed between the fission sources in the two cores when they were decoupled by concrete. This behavior is demonstrated in the " 30 $\mathrm{cm}$ " trace in Figure 2. The authors' analysis of mechanisms driving this behavior is interesting and illuminating, but we will not repeat it in detail. For our purposes it is sufficient to say that fluctuations are injected into the MC computation within each generation, and are weakened because leakage tends to smear irregularities in the both the source shape and the flux shape. The rate of attenuation depends on the dominance ratio; if it is close to unity the fluctuations may grow to very large magnitudes until an equilibrium is attained between the rate of creation and attenuation of noise.

In this section we have two purposes. First we want to compare the efficiency of the superhistory method with that of conventional MC, as in earlier sections; but now in addition we would like to improve our understanding of the observed anomalous behavior and, as far as we can, to damp out the erratic shifts in power shapes.

Since, as we have already pointed out, we have not implemented a full-scale ANL superhistory code, our superhistory computations will again be run as one-dimensional onegroup slabs using a special test code. Group constants for these computations were produced by VIM in the course of continuous-energy MC runs for the JAERI two-core configuration. Only the symmetric version of this problem will be discussed here. For convenience, and to facilitate comparisons with the one-group calculations, the VIM calculations were run as one-dimensional slabs. Further, we have replaced the $30 \mathrm{~cm}$ slab of concrete with $30 \mathrm{~cm}$ of water. All relevant problem characteristics are listed in Tables III and IV, and corresponding computational results are tabulated in Table V. As throughout this paper, the "variances" cited are the variances among 
the average absorption rates estimated in individual replicas, i.e., not the average of the variances estimated within the replicas. Thus, to the extent that the replicas are independent, the variance computations are rigorous.

As before, it will be seen that, in this case, there is little difference in efficiency between conventional and superhistory $\mathrm{MC}$; variances in absorption rates differ little (perhaps insignificantly) between methods. At this stage, the fact that absorption rates are much more symmetric in the superhistory calculation would, therefore, seem to be accidental. On the other hand there is a large difference in absorption rate variances in test cases 3 and 4, indicating a large disadvantage in the use of collision sites as potential fission sites.

Because of the anomalous behavior of the MC results in the JAERI problem, and because the JAERI problem is a system encountered in practice, it was felt to be particularly interesting to run more realistic multi-energy test computations. For this purpose a VIM version was written in which absorption sites, instead of collision sites, were taken as potential source sites. To check the consistency of methods between VIM and in the specialized test codes (methods which are not identical even for the 1D one-group test problems), the one-group tests were rerun with VIM. Results of this computation are displayed as Test Case 4 in Table V. These results, in view of the relatively small number of replicas, seem consistent with those shown above for the corresponding computation. VIM continuous-energy solution variances for the JAERI problem, again for 20 replicas using each fission site generation scheme, are shown in Tables VI and VII.

Comparing one-group and continuous-energy results, it's clear that the one-group model is too unrealistic to evaluate the site generation techniques accurately. This can be seen both by comparing variances, and absorption rates in region 2. One reason for the observed differences may be the fact that there is, in water, a cross section window at high energies, a window through which neutrons can pass from core to core much more easily than in the one-group model. Surely another reason is that there are many sources of statistical noise in the realistic VIM that are absent in one-group MC. At this point we cannot say that all the differences in variance are thoroughly understood. In any case, variances in the VIM absorption rates are about 2.6 times as large when collision sites are taken as source sites as they are when absorption sites are taken as source sites. Since running times are about the same for both problems, we conclude that the efficiency improves by a factor of about 2.6 when absorption sites are taken as potential source sites.

This difference in variance is easily explained qualitatively in the limiting case of infinitely large cores. In this limit the motion of neutrons is unimportant. One can assume, in fact, that neutrons don't move at all between collisions. Suppose that starters are injected into this configuration at the beginning of a generation. Assume that the absorption probability is small, so that each starter makes a large number of collisions before it is absorbed. The number of collisions then has a large variance. Each collision site is assigned a weight of $\mathrm{v} \Sigma_{\mathrm{f}} /\left(\mathrm{k} \Sigma_{\mathrm{t}}\right)$, which is usually much less than unity. This weight is then used as a basis for Russian roulette. Thus, for example, a starter might produce, say, 50 collision sites, which are then rouletted back to about one new starting site. The roulette process also produces noise, and the added variance propagates from one generation and one supergeneration to the next. In a finite medium this accumulation of variance would be counteracted by neutron transport from regions of high 
neutron density to regions of lower density, but would be enhanced by the fact that sites from the same neutron track are not independent source sites.

These considerations are treated quantitatively in Appendices A and B, the first two of four appendices. In these two Appendices we compute the variances in fission rates in each core using random source-resampling, in A taking potential source sites at absorption sites, and in B using collision sites. In both cases the variances increase linearly with the number of generations (assuming, in B, that fluctuations are small); but if the ratio of scattering to total cross sections is close to unity the added variance due to the use of collision sites is equal to the variance due to random source-resampling. It appears, from the numerical results, that the random sourceresampling tends to smear the source fluctuations over both cores. If random source-resampling were replaced by a less noisy population control mechanism this smearing might be weakened. The variance injected directly by population control would then decrease, but that due to the use of collision sites might increase. Further, it seems plausible, from results obtained in Appendix $\mathrm{B}$, that without population control (as in the superhistory method) the variance due to the use of collision sites might double, but this possibility has not yet been investigated.

It is interesting to see how the added variance affects the ratio, $\mathrm{r}$, of fission rates $S f$, in the two cores. In Figure 3 we have plotted the greater, r rax, of the ratios $S f_{2} / S f_{1}$ and $S f_{1} / S f_{2}$ for all 20 replicas, and for runs with both schemes for constructing source sites. In this plot, replicas are ordered by increasing values of $r_{\max }$. Generally $r_{\max }$ is about 2.5 times greater for the collisionbased algorithm than for the absorption-based algorithm. Further, two replicas using the collision based source selection algorithm give bizarrely large values (about 100) for $r_{\max }$; in the computations using absorption site-based starting sites no ratio is greater than 5 .

Appendices $\mathrm{C}$ and $\mathrm{D}$ have been added in the hope that they, together with $\mathrm{A}$ and $\mathrm{B}$, will give some added insight into the behaviour of variances in the symmetric coupled core problem. In $\mathrm{C}$ we show that, very generally, again for infinitely large cores and in the limit of small fluctuations, the variances in fission rates, per core per generation, increase linearly with the number of generations. Further, the variances in average-over-generations fission rates also increase linearly as the number of generations goes to infinity. As one might expect from the arguments of Ref. 5, this behaviour of variances is not sensitive to the details of the Monte Carlo process. Finally, in Appendix D we consider the effect of coupling the two cores, though only in a two-point model (i.e., a model in which each core is represented as a point) and in the limit of small fluctuations. In this case any nonzero coupling will make the variances in the pergeneration fission source go to a finite limit as the number of generations increases and, correspondingly, the variances in the means-over-generations will go to zero like the inverse of the number of generations. However, as the core coupling becomes weaker, then, for a given number of starting neutrons, fluctuations will grow from one generation to another till the assumption of small fluctuations will fail and Monte Carlo biases may then become significant. Just at what point biases appear can be determined most easily directly from a superhistory calculation. 


\section{Large LWR Core}

A coupled two-core reactor configuration is still unusual, but it is important to realize that $\mathrm{MC}$ results for much more common reactor configurations may also exhibit anomalies. In this section we deal with MC computations for two large thermal reactor cores, the first homogenized and the second composed of slab subassemblies. Both cores have vacuum boundaries.

The homogeneous "reactor" consisted of a $35 \times 35$ array of square units, $350 \mathrm{~cm}$ on a side and $350 \mathrm{~cm}$ in length. Each unit is a volumetrically smeared PWR subassembly, with smeared B10 added to achieve criticality at $300(\mathrm{~K})$. The boron density was increased in the fourth (17x17) quadrant to introduce the effects of a flux tilt. Atom densities are shown in Table VIII.

Tables IX-A and IX-B list VIM continuous-energy computational results for 20 replicas, 4000 neutrons per generation, 400 generations, 0 skipped, using absorptions and collisions, respectively, to produce potential fission sites. Quadrant 1 is in the upper left-hand corner, quadrant 4 is in the lower right-hand corner (with extra boron), and 2 and 3 are the remaining quadrants.

It will be seen from Tables IX-A and IX-B that the pattern of MC results is similar to the pattern observed in the JAERI problem; i.e. that the computed fission production rates are significantly more noisy when source sites are taken at collision sites then when they are taken at absorption sites. In both cases, the "variances" computed among generations of a single run are extremely inaccurate, but are substantially worse when collision sites are used as potential fission sites.

Our final problem is a hypothetical heterogeneous version of the previous homogeneous problem. In this case the reactor fuel and structural materials are collected into $0.5 \mathrm{~cm}$ plates, while the boron and moderator are contained in separate moderator regions, also $0.5 \mathrm{~cm}$ thick. Atom densities are listed below in Table X, while computational results with the absorptionbased (case A) and collision-based (case B) algorithms are displayed in Tables XI-A and XI-B, respectively.

In this case, much of the advantage of the use of absorption sites as potential collision sites has been lost. The reason is pretty clear. Here the fission sites appear in regions where the ratio of scattering to absorption cross sections is relatively small, so that the number of potential fission sites per starter is also relatively small. The variance involved in producing and rouletting the potential source sites is then also small compared to the variance produced in the homogeneous case. It will be seen that the "apparent variances" computed among generations are now somewhat more accurate in both cases, and are not much better, if at all, in case A than in case B.

\section{CONCLUSIONS}

In all of the one-group test problems the superhistory method and conventional $\mathrm{MC}$ are equally efficient. Fluctuations in the numbers of source neutrons do accumulate within each supergeneration. On the other hand since eigenvalues are defined as numbers of fission neutrons 
produced per neutron absorbed, these fluctuations don't affect eigenvalue estimates. Local fluctuations in source shapes also accumulate in the superhistory method, but intergeneration normalizations in conventional MC don't prevent the same sort of noise accumulation, in part a result of intergeneration correlations. Such correlations can be weakened by normalization between generations only if it introduces its own additional noise. Thus in both the eigenvalue computation and in the estimation of local reaction rates, noise will accumulate at much the same rate in the superhistory method and in conventional MC.

It must be stressed that we are referring here only to test problems of types discussed above. In the Eigenvalue of the World problem anomalies are caused primarily by the loss of source regions during the normalization process. The frequency of normalizations is reduced in the superhistory method, and the frequency of anomalous MC results is thereby reduced. Performances of the superhistory method and of stratified source sampling (Ref. 8) in Eigenvalue of the World problems are compared in Ref. 9. Stratified source sampling relies for success on reducing the likelihood of losing source regions via normalizations, while the superhistory method achieves the same goal by reducing the normalization frequency. At any rate, there seems to be no disadvantage connected with the use of the superhistory method; apparently no cost is incurred in substantially weakening biases.

In eigenvalue calculations for configurations with large cores it is sometimes preferable to take potential source sites at absorption sites, rather than collision sites. This seems to be particularly true with systems in which many scatterings per starter occur in important multiplying media.

In the work reported here we have seen no case where it is preferable to use collision sites as potential source sites. Yet on an intuitive basis many (perhaps most) Monte Carlo specialists have preferred this option. In using only the absorption sites we seem to discard much of the information contained in the neutron histories. On the other hand, selection of source sites from collision sites can be a very noisy process and, furthermore, source sites taken from the same track, or even the same history, are dependent samples whose lack of independence may seriously impair their value. Thus in a very large region the length of a track, or even the net displacement of a neutron during its history, may be insignificant compared to the dimensions of the region. In such a case it could be relatively inefficient to track more than one starter from the same history.

Thus it is difficult, on purely theoretical grounds, to come to a generally valid conclusion as to which of these two site-generation schemes should be used. In fact there may be cases where (for small numbers of histories per generation) the chain reaction terminates within some region when sources are taken at absorption sites, but not when they are taken at collision sites. At this point, it isn't clear that one method or the other should always be preferred, but it is true that the use of collision sites to generate potential fission source sites isn't always the best strategy. 


\section{REFERENCES}

1. R. J. BRISSENDEN and A. R. GARLICK, "Biases in the Estimation of $\mathrm{k}_{\text {eff }}$ and its Error by Monte Carlo Methods," Ann. Nucl. Energy, Vol. 113, No. 2, pp. 63-83 (1986).

2. E. GELBARD and A. GU, "Biases in Monte Carlo Eigenvalue Calculations," Nucl. Sci. and Eng., Vol. 117, pp. 1-9 (1994).

3. N. SMITH et al., "The Unification of MONK, Extending the Monte Carlo Horizon," Sixth International Conference on Nuclear Criticality Safety, Vol. 1, pp. 38-47, Versailles, FR, September 20-24, 1999.

4. J. F. BRIESMEISTER, Editor, "MCNP A General Monte Carlo N-Particle Transport Code," Version 4A, Los Alamos National Laboratory (1993).

5. T. YAMAMOTO, et al., "Fission Source of Monte Carlo Criticality Calculations in Weakly Coupled Fissile Arrays," J. of Nucl. Sci. and Tech., Vol. 37, No. 1, pp. 41-45 (2000).

6. R. N. BLOMQUIST, "VIM-A Continuous Energy Monte Carlo Code at ANL, A Review of the Theory and Application of Monte Carlo Method", Proceedings of a SeminarWorkshop, ORNL/RSIC-44 (April 1980).

7. T. C. Booth, "A Weight (Charge) Conserving Importance-Weighted Comb for Monte Carlo". Proceedings of the 1996 Radiation Protection and Shielding Topical Meeting, North Falmouth, MA, April 21-25, 1996.

8. A. MOHAMED and E. GELBARD, "Stratified Source-Sampling Techniques for Monte Carlo Eigenvalue Analysis," Proc. Int. Conf. On Physics of Nuclear Science and Technology, Hauppage, N.Y., Oct. 5-8, 1998.

9. R. N. BLOMQUIST and E. M. GELBARD, "Monte Carlo Source Convergence and the Whitesides Problem", Proc PHYSOR 2000, II-A-3, Pittsburgh, PA, May 7-12, 2000. 

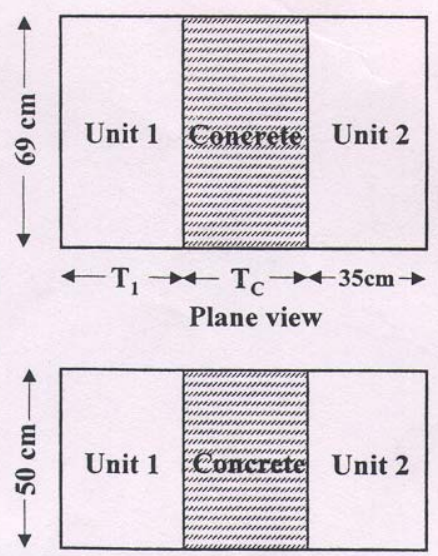

Elevation view

Figure 1. Schematic View of JAERI Coupled System (Ref. 5, reproduced with permission)

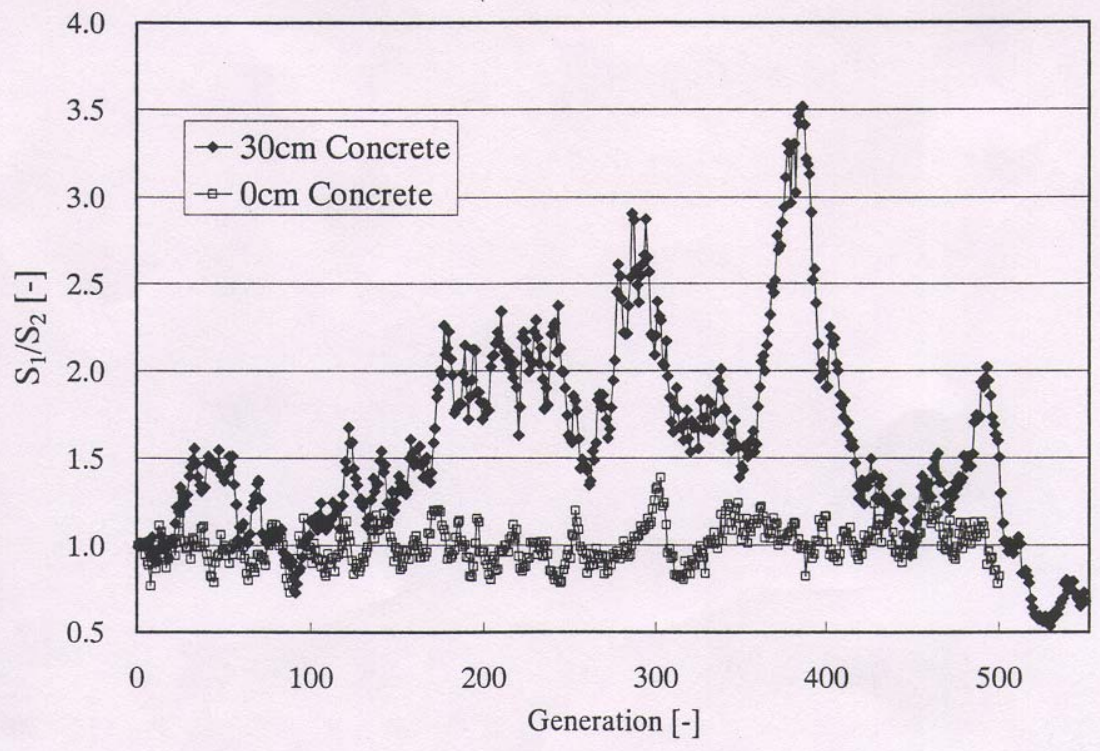

Figure 2. Ratio of Starting Neutrons (Slab 1/Slab 2) in Each Generation $\left(T_{1},=35,2000\right.$ Neutrons/Generation) (Ref. 5, reproduced with permission) 


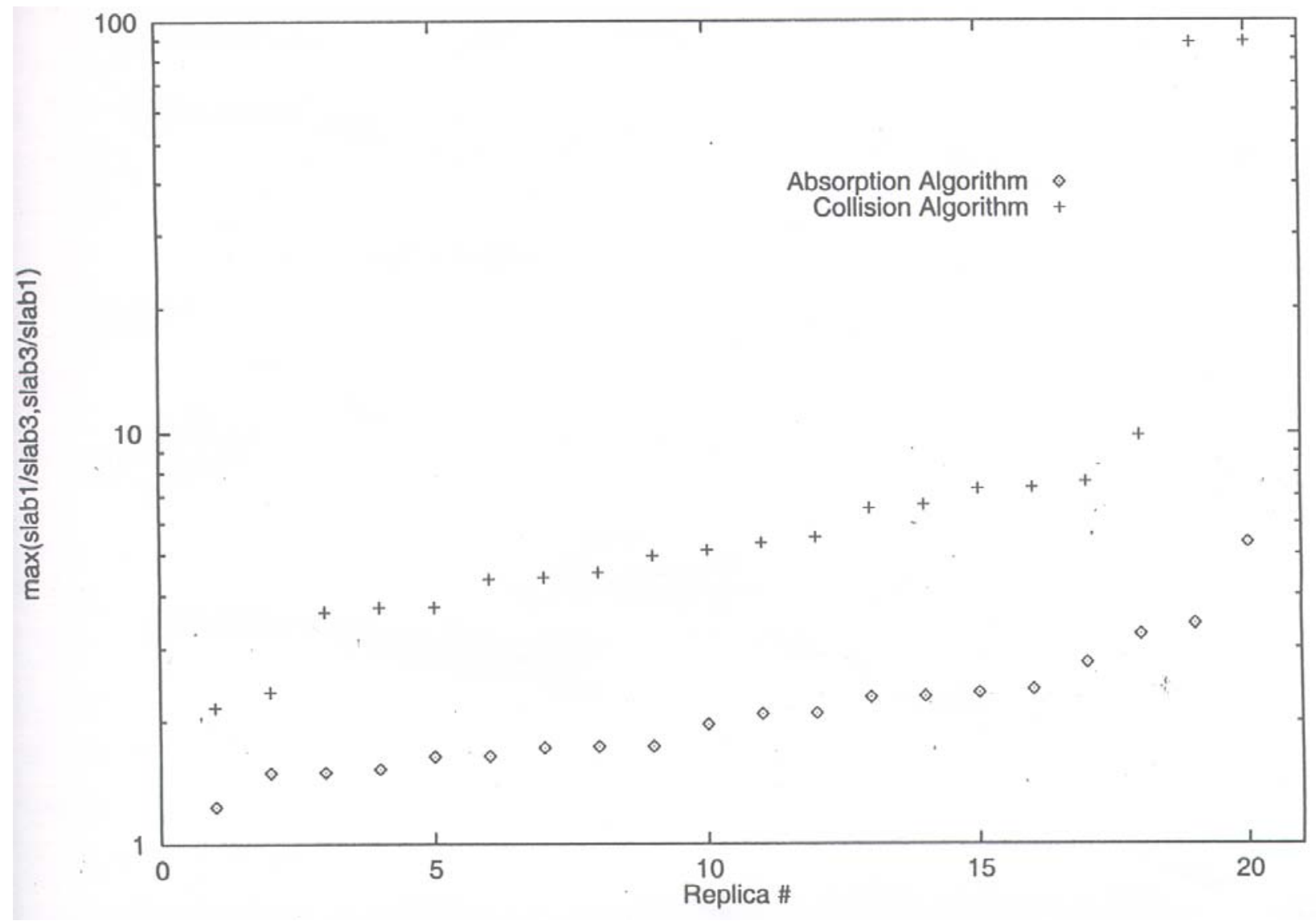

Figure 3. JAERI Slabs: Maximum Fission Ratios for 20 Replicas, Ordered by Increasing Ratio. 
TABLE I.

Absorption and $k_{\text {eff }}$ Variances for Three Uniform Slabs (One-Group) ${ }^{a}$

\begin{tabular}{|c|c|c|c|c|c|c|}
\hline Method, Variant & $\Sigma_{\mathrm{f}}$ & $\overline{\mathrm{v}}$ & $\begin{array}{l}\text { Generations/ } \\
\text { Superhistory }\end{array}$ & $\begin{array}{c}\text { Number of } \\
\text { Superhists }\end{array}$ & $\sigma_{\mathrm{a}}{ }^{2}$ & $\sigma_{\mathrm{k}}{ }^{2}$ \\
\hline Conventional, E = A & 0.1 & 1.0 & 1 & 100 & 6.06 & $1.8 \mathrm{e}-5$ \\
\hline Superhistory, A & $0.1 / \overline{\mathrm{v}}$ & 3.0 & 10 & 10 & 9.28 & $1.7 \mathrm{e}-5$ \\
\hline Superhistory, E = A & 0.1 & 1.0 & 10 & 10 & 3.92 & $1.7 \mathrm{e}-5$ \\
\hline
\end{tabular}

${ }^{a}$ Reflecting Boundaries, $\Sigma_{\mathrm{t}}=1 ., \Sigma_{\mathrm{a}}=0.1 ;$ run with special test code.

TABLE II.

Absorption Rate Variances, $k_{\text {eff }}$ and Variances in Non-Uniform Slabs (One-Group): No ReSampling.

\begin{tabular}{|c|c|c|c|c|c|c|c|}
\hline \multirow{2}{*}{$\begin{array}{c}\text { Problem } \\
\text { Set }\end{array}$} & Method/ & \multicolumn{2}{|c|}{$\sigma_{\mathrm{a}}^{2}$} & \multicolumn{2}{c|}{ Eigenvalue, $\mathrm{k}$} & \multicolumn{2}{c|}{$\sigma_{\mathrm{k}}{ }^{2}$} \\
\hline 1 & Variant $^{\mathrm{a}}$ & $\overline{\mathrm{v}}=3.0$ & $\overline{\mathrm{v}}=2.5$ & $\overline{\mathrm{v}}=3.0$ & $\overline{\mathrm{v}}=2.5$ & $\overline{\mathrm{v}}=3.0$ & $\overline{\mathrm{v}}=2.5$ \\
1 & Conventional & 3.86 & 3.86 & .99993 & .99993 & $1.63 \mathrm{e}-5$ & $1.63 \mathrm{e}-5$ \\
1 & Superhist./A & 9.52 & 7.94 & .99984 & .99973 & $1.78 \mathrm{e}-5$ & $1.90 \mathrm{e}-5$ \\
\hline 2 & Superhist./E & 3.64 & 3.64 & .99993 & .99993 & $1.63 \mathrm{e}-5$ & $1.63 \mathrm{e}-5$ \\
2 & Conventional & 2.71 & 2.71 & .77045 & .77045 & $1.04 \mathrm{e}-5$ & $1.04 \mathrm{e}-5$ \\
2 & Superhist./A & 4.99 & 4.09 & .77000 & .77030 & $1.37 \mathrm{e}-5$ & $1.28 \mathrm{e}-5$ \\
3 & Superhist./E & 2.46 & 2.46 & .77024 & .77024 & $1.04 \mathrm{e}-5$ & $1.04 \mathrm{e}-5$ \\
\hline 3 & Conventional & 1.45 & 1.45 & .69191 & .69191 & $9.64 \mathrm{e}-6$ & $9.64 \mathrm{e}-6$ \\
3 & Superhist./A & 1.68 & 1.55 & .69201 & .69185 & $9.56 \mathrm{e}-6$ & $9.27 \mathrm{e}-6$ \\
& Superhist./E & 1.51 & 1.51 & .69190 & .69190 & $9.64 \mathrm{e}-6$ & $9.64 \mathrm{e}-6$ \\
\hline
\end{tabular}

${ }^{a}$ Runs replicated 500 times with a special test code. Variances were computed in each region, then averaged over regions to give table entries. In all regions $\Sigma_{\mathrm{a}}=.1$ and $\Sigma_{\mathrm{t}}=1.0$. Problems in set 1 are physically the same as problem of Table I.

TABLE III.

JAERI Problem, One-Group Parameters ${ }^{\text {a }}$

\begin{tabular}{|l|l|l|l|l|}
\hline Region & $\Sigma_{\mathrm{a}}\left(\mathrm{cm}^{-1}\right)$ & $\Sigma_{\mathrm{s}}\left(\mathrm{cm}^{-1}\right)$ & $\Sigma_{\mathrm{f}}\left(\mathrm{cm}^{-1}\right)$ & $\mathrm{T}(\mathrm{cm})$ \\
\hline 1 & 0.0256 & 1.5600 & 0.0135 & 35.0 \\
\hline 2 & 0.0135 & 2.5300 & 0.0 & 30.0 \\
\hline 3 & 0.0256 & 1.5600 & 0.0135 & 35.0 \\
\hline
\end{tabular}

${ }^{\text {a }}$ In all regions $\overline{\mathrm{V}}=2.44$. 
TABLE IV.

\section{Computational Characteristics: One-Group JAERI Problem}

\begin{tabular}{|c|c|c|c|}
\hline $\begin{array}{c}\text { Test } \\
\text { Case }\end{array}$ & $\begin{array}{c}\text { Generations per } \\
\text { Supergeneration }\end{array}$ & $\begin{array}{c}\text { Number of } \\
\text { Supergenerations }\end{array}$ & $\begin{array}{c}\text { Potential Fission } \\
\text { Source-sites at: }\end{array}$ \\
\hline 1 & 1 & 400 & Absorption \\
\hline 2 & 10 & 40 & Absorption \\
\hline 3 & 1 & 400 & Collision \\
\hline 4 (VIM) & 1 & 400 & Absorption \\
\hline
\end{tabular}

TABLE V.

JAERI Problem: One-Group Monte Carlo Results ${ }^{\text {a }}$

\begin{tabular}{|c|c|c|c|c|c|c|}
\hline & \multicolumn{3}{|c|}{ Absorption Rates } & \multicolumn{3}{c|}{ Absorption Rate Variances } \\
\hline Test Case & Region 1 & Region 2 & Region 3 & Region 1 & Region 2 & Region 3 \\
\hline 1 & 959.4 & 46.1 & 944.5 & 6357. & 0.2 & 6357. \\
\hline 2 & 951.6 & 46.0 & 952.3 & 6210. & 0.2 & 6242. \\
\hline 3 & 919.5 & 46.1 & 984.4 & 95377. & 1.1 & 95455. \\
\hline 4 (VIM) & 940.4 & 46.0 & 963.6 & 5544. & 0.1 & 5536. \\
\hline
\end{tabular}

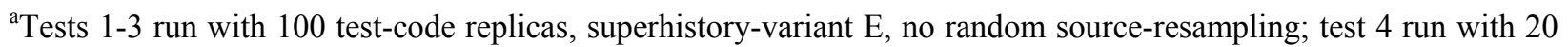
VIM replicas.

TABLE VI.

JAERI Problem: VIM Continuous-Energy Results, Collision Site Technique

\begin{tabular}{|c|c|c|c|}
\hline Region: & 1 & 2 & 3 \\
\hline Absorption Rates & 857.593 & 134.374 & 871.263 \\
\hline Variance & 48856.7 & 1.3 & 48805.9 \\
\hline
\end{tabular}

TABLE VII.

JAERI Problem: VIM Continuous-Energy Results, Absorption Site Technique

\begin{tabular}{|c|c|c|c|}
\hline Region: & 1 & 2 & 3 \\
\hline Absorption Rates & 860.844 & 134.549 & 867.934 \\
\hline Variance & 19080.5 & 0.5 & 19080.3 \\
\hline
\end{tabular}


TABLE VIII.

Homogeneous LWR Atom Densities

\begin{tabular}{|c|c|}
\hline Nuclide & Density $(\mathrm{at} / \mathrm{b}-\mathrm{cm})$ \\
\hline $\mathrm{U}-235$ & $1.93800 \mathrm{E}-04$ \\
\hline $\mathrm{U}-238$ & $6.26732 \mathrm{E}-03$ \\
\hline O-16 & $3.3398 \mathrm{E}-02$ \\
\hline B-10 (quadrants 1-3) & $1.2500 \mathrm{E}-05$ \\
\hline B-10 (quadrant 4) & $1.5000 \mathrm{E}-05$ \\
\hline Zirc & $4.1949 \mathrm{E}-03$ \\
\hline H & $4.0952 \mathrm{E}-02$ \\
\hline
\end{tabular}

TABLE IX-A.

Homogeneous LWR Results: Continuous Energy Absorption-Sites as Potential Source-Sites

\begin{tabular}{|c|c|c|c|c|}
\hline Parameter & Quad 1 & Quad 2 & Quad 3 & Quad 4 \\
\hline Avg. Fission Production & 1554.1 & 1135.1 & 1118.3 & 269.3 \\
\hline Variance (20 replicas) & 13415.1 & 15263.0 & 13900.8 & 530.8 \\
\hline Std. Dev. of Mean (20 replicas) & 26.6 & 28.3 & 27.0 & 5.3 \\
\hline Avg. of Std. Dev. Estimates & 9.4 & 6.6 & 6.2 & 6.6 \\
\hline
\end{tabular}

TABLE IX-B.

Homogeneous LWR Results: Continuous Energy, Collision-Sites as Potential Source-Sites

\begin{tabular}{|c|c|c|c|c|}
\hline Parameter & Quad 1 & Quad 2 & Quad 3 & Quad 4 \\
\hline Avg. Fission Production & 1474.0 & 1224.0 & 1095.1 & 2283.9 \\
\hline Variance (20 replicas) & 31248.1 & 81030.9 & 102066.2 & 1568.4 \\
\hline Std. Dev. of Mean (20 replicas) & 40.6 & 65.3 & 733.3 & 9.1 \\
\hline Avg. of Std. Dev. Estimates & 14.3 & 13.4 & 12.7 & 7.8 \\
\hline Var.(IX-B)/Var.(IX-A) & 2.3 & 5.3 & 7.34 & 3.0 \\
\hline
\end{tabular}


TABLE X.

Heterogeneous LWR Atom Densities

\begin{tabular}{|c|c|c|}
\hline Plate & Nuclide & Density (at/b-cm) \\
\hline Fuel & U-235 & $3.87600 \mathrm{E}-04$ \\
\hline Fuel & U-238 & $1.25346 \mathrm{E}-02$ \\
\hline Fuel & O-16 & $2.58444 \mathrm{E}-02$ \\
\hline Fuel & Zirc & $8.3898 \mathrm{E}-03$ \\
\hline Moderator & O-16 & $3.3330 \mathrm{E}-02$ \\
\hline Moderator & B-10 (quadrants 1-3) & $2.5000 \mathrm{E}-05$ \\
\hline Moderator & B-10 (quadrant 4) & $2.7500 \mathrm{E}-05$ \\
\hline Moderator & H & $6.6660 \mathrm{E}-02$ \\
\hline
\end{tabular}

TABLE XI-A.

Heterogeneous LWR Results: Continuous Energy, Absorption Sites as Potential Fission Sites ${ }^{\mathrm{a}}$

\begin{tabular}{|c|c|c|c|c|}
\hline Parameter & Quad 1 & Quad 2 & Quad 3 & Quad 4 \\
\hline Avg. Fission Production & 1406.48 & 1070.23 & 1102.09 & 519.584 \\
\hline Variance (20 replicas) & 5253.99 & 11177.8 & 11181.1 & 1465.37 \\
\hline Std. dev. of mean (20 replicas) & 16.6291 & 24.2551 & 24.2585 & 8.78206 \\
\hline Avg. of std. dev. estimates & 12.0663 & 7.47985 & 7.14026 & 13.1324 \\
\hline
\end{tabular}

${ }^{\mathrm{a}} \mathrm{VIM}$ continuous energy. 20 replicas, 4000 neutrons, 400 generations ( 0 skipped)

TABLE XI-B.

Heterogeneous LWR Results: Continuous Energy, Collision Sites as Potential Fission Sites ${ }^{\mathrm{a}}$

\begin{tabular}{|c|c|c|c|c|}
\hline Parameter & Quad 1 & Quad 2 & Quad 3 & Quad 4 \\
\hline Avg. Fission Production & 1418.71 & 1041.47 & 1124.86 & 514.082 \\
\hline Variance (20 replicas) & 14125.6 & 9837.27 & 9180.52 & 3052.65 \\
\hline Std. dev. of mean (20 replicas) & 27.2663 & 22.7541 & 21.9815 & 12.6754 \\
\hline Avg. of std. dev. estimates & 11.9085 & 8.50203 & 9.15421 & 12.9712 \\
\hline
\end{tabular}

${ }^{a}$ VIM continuous energy. 20 replicas, 4000 neutrons, 400 generations ( 0 skipped) 


\section{Appendix A}

\section{Theory of Variance \\ Due to Random Source-Resampling in Eigenvalue Calculations}

In this Appendix we compute the variance induced in fission distributions by intergeneration source-resampling, the process specified earlier in Fortran notation. We do this so that, in a later Appendix, we can compare this variance with variances introduced by the use of collision sites as potential source sites. To make such a comparison analytically, it will be necessary to confine our attention to the simplest configuration which still retains some interest. Here this is a system of two media, both homogeneous and very large, in a one-group model. We assume, in fact, that the media are so large that within them the displacements of moving neutrons are negligibly small. The two media can be different parts of one single medium, but parts so large that interactions between them may be neglected. In our case, both media have the same cross sections and are both just critical. One occupies a region, $R$, whose volume is a fraction, $p$, of the total volume of both. We focus attention on $R$. Suppose that the source guess is flat and that $N_{l}$ starters for the first generation are sampled from this source. In this case, the probability that a starter falls into $R$ is $p$. Let $n_{l}$ be the number of starters in $R$ in the current generation, and $N_{2}$ the total number of starters we need for the next generation. We want to compute the variance in $n_{2}$, the number of next-generation starters in $R$. In the following discussion, we define $P_{1}\left(n_{1}\right)$ to be the probability that $n_{1}$ starters begin in $R, P_{2}\left(n_{2}\right)$ to be the probability that $n_{2}$ next-generation starters are in $R$, and $P_{21}\left(n_{2} \mid n_{1}\right)$ to be the probability that $n_{2}$ next-generation starters are in $R$ given that $n_{l}$ neutrons start in $R$.

In our one-group model of the JAERI problem, the eigenvalue, $\mathrm{k}_{0}$, is equal to $\mathrm{k}_{\text {inf, }}$, i.e., $\mathrm{k}_{0}$ $=\bar{v} \Sigma_{\mathrm{f}} / \Sigma_{\mathrm{a}}$. If, as we assume here, absorption sites are taken as potential fission sites, the site weights, w, are equal to $\bar{v} \Sigma_{\mathrm{f}} /\left(\mathrm{k} \Sigma_{\mathrm{a}}\right)$. Suppose that here as in all our one-group problems, we take $\mathrm{k}=\mathrm{k}_{0}$ so that $\mathrm{w}=1$. Then in Variant $\mathrm{E}$, exactly one fission neutron is emitted at each absorption site. Essentially, neutrons are passing directly from the source into the source-resampling process.

Now, by definition of the variance of $n_{2}$ during the MC calculation, and using

$$
\begin{aligned}
P_{2}\left(n_{2}\right) & =\sum_{n_{1}} P_{21}\left(n_{2} \mid n_{1}\right) P_{1}\left(n_{1}\right), \\
\sigma^{2}\left(n_{2}\right) & =\sum_{n_{2}}\left(n_{2}-\bar{n}_{2}\right)^{2} P_{2}\left(n_{2}\right) \\
& =\sum_{n_{1}} \sum_{n_{2}}\left[n_{2}-\left(\bar{n}_{2} \mid n_{1}\right)+\left(\bar{n}_{2} \mid n_{1}\right)-\bar{n}_{2}\right]^{2} P_{21}\left(n_{2} \mid n_{1}\right) P_{1}\left(n_{1}\right) .
\end{aligned}
$$

Here $\left(\bar{n}_{2} \mid n_{1}\right) \equiv \sum_{n_{2}} n_{2} P_{21}\left(n_{2} \mid n_{1}\right)$, i.e. $\left(\bar{n}_{2} \mid n_{1}\right)$ is the conditional mean of $\mathrm{n}_{2}$ given $\mathrm{n}_{1}$. Expanding the square, Eq. (1) becomes 


$$
\begin{aligned}
\sigma^{2}\left(n_{2}\right) & =\sum_{n_{1}} \sum_{n_{2}}\left[n_{2}-\left(\bar{n}_{2} \mid n_{1}\right)\right]^{2} P_{21}\left(n_{2} \mid n_{1}\right) P_{1}\left(n_{1}\right) \\
& +\sum_{n_{1}}\left[\left(\bar{n}_{2} \mid n_{1}\right)-\bar{n}_{2}\right]^{2} P_{1}\left(n_{1}\right) \\
& +2 \sum_{n_{1}} \sum_{n_{2}}\left[n_{2}-\left(\bar{n}_{2} \mid n_{1}\right)\right]\left[\left(\bar{n}_{2} \mid n_{1}\right)-\bar{n}_{2}\right] P_{21}\left(n_{2} \mid n_{1}\right) P_{1}\left(n_{1}\right),
\end{aligned}
$$

where the second term on the right uses the fact that $\sum_{n_{2}} P_{21}\left(n_{2} \mid n_{1}\right)=1$. In abbreviated notation, we write

$\sigma^{2}\left(n_{2}\right)=\sigma_{1}^{2}\left(n_{2}\right)+\sigma_{2}^{2}\left(n_{2}\right)+\sigma_{3}^{2}\left(n_{2}\right)$

where we define the three variance components below:

$$
\begin{aligned}
& \sigma_{1}^{2}\left(n_{2}\right) \equiv \sum_{n_{1}} \sum_{n_{2}}\left[n_{2}-\left(\bar{n}_{2} \mid n_{1}\right)\right]^{2} P_{21}\left(n_{2} \mid n_{1}\right) P\left(n_{1}\right), \\
& \sigma_{2}^{2}\left(n_{2}\right) \equiv \sum_{n_{1}}\left[\left(\bar{n}_{2} \mid n_{1}\right)-\bar{n}_{2}\right]^{2} P_{1}\left(n_{1}\right) \\
& \sigma_{3}^{2}\left(n_{2}\right) \equiv 2 \sum_{n_{1}} \sum_{n_{2}}\left[n_{2}-\left(\bar{n}_{2} \mid n_{1}\right)\right]\left[\left(\bar{n}_{2} \mid n_{1}\right)-\bar{n}_{2}\right] P_{21}\left(n_{2} \mid n_{1}\right) P_{1}\left(n_{1}\right) .
\end{aligned}
$$

Next, we show that $\sigma_{3}^{2}\left(n_{2}\right)=0$. It will be seen that $\left[\left(\bar{n}_{2} \mid n_{1}\right)-\bar{n}_{2}\right]$ depends only on $\mathrm{n}_{1}$, since it has already been averaged over $n_{2}$ keeping $n_{1}$ fixed. Therefore, one can write

$$
\sigma_{3}^{2}\left(n_{2}\right)=\sum_{n_{1}}\left\{\sum_{n_{2}}\left[n_{2}-\left(\bar{n}_{2} \mid n_{1}\right)\right] P_{21}\left(n_{2} \mid n_{1}\right)\right\}\left[\left(\bar{n}_{2} \mid n_{1}\right)-\bar{n}_{2}\right] P_{1}\left(n_{1}\right)
$$

But by definition $\left(\bar{n}_{2} \mid n_{1}\right)=\sum_{n_{2}} n_{2} P_{21}\left(n_{2} \mid n_{1}\right)$, so that in Eq. (4), the quantity in curly brackets vanishes. Thus, we are left with

$$
\sigma^{2}\left(n_{2}\right)=\sigma_{1}^{2}\left(n_{2}\right)+\sigma_{2}^{2}\left(n_{2}\right) .
$$

The computation of $\sigma_{2}^{2}$ is relatively simple, so we dispose of this computation first. If $n_{1}$ is given, the population of $N_{l}$ fission sites contains a fraction $n_{l} / N_{l}$ of sites within $R$. In each resampling step, this ratio is also the probability of choosing a next-generation starting site in $R$. Therefore,

$$
\left(\bar{n}_{2} \mid n_{1}\right)=N_{2} n_{1} / N_{1}
$$

Further, $\bar{n}_{2}=\sum_{n_{1}}\left(\bar{n}_{2} \mid n_{1}\right) P_{1}\left(n_{1}\right)$. Because $N_{l}$ and $N_{2}$ are constant, and from Eq. (3) and the two preceding equations, we see that 


$$
\begin{aligned}
\sigma_{2}^{2}\left(n_{2}\right) & =\sum_{n_{1}}\left(N_{2} n_{1} / N_{1}-N_{2} \bar{n}_{1} / N_{1}\right)^{2} P_{1}\left(n_{1}\right) \\
& =\left(N_{2} / N_{1}\right)^{2} \sum_{n_{1}}\left(n_{1}-\bar{n}\right)^{2} P_{1}\left(n_{1}\right), \\
\sigma_{2}^{2}\left(n_{2}\right) & =\left(N_{2} / N_{1}\right)^{2} \sigma^{2}\left(n_{1}\right),
\end{aligned}
$$

where

$$
\bar{n}_{1}=\sum_{n_{1}} n_{1} P_{1}\left(n_{1}\right)
$$

Because we have a binomial distribution of starters,

$$
\sigma_{2}^{2}\left(n_{2}\right)=\left(N_{2}^{2} / N_{1}\right) p(1-p)
$$

The computation of $\sigma_{1}^{2}\left(n_{2}\right)$ is only slightly more complicated. For this case, there are $n_{2}$ starters, each with a binomial distribution with probability $p=n_{1} / N_{l}$, i.e.,

$$
\sum_{n_{2}}\left[n_{2}-\left(\bar{n}_{2} \mid n_{1}\right)\right]^{2} P_{21}\left(n_{2} \mid n_{1}\right)=N_{2}\left(\frac{n_{1}}{N_{1}}\right)\left[1-\left(\frac{n_{1}}{N_{1}}\right)\right] \text {, }
$$

so that the first of Eqs. (3) becomes

$$
\begin{aligned}
\sigma_{1}^{2}\left(n_{2}\right) & =\sum_{n_{1}} N_{2}\left(\frac{n_{1}}{N_{1}}\right)\left[1-\left(\frac{n_{1}}{N_{1}}\right)\right] P_{1}\left(n_{1}\right) \\
& =N_{2}\left(p-\frac{\overline{n_{1}^{2}}}{N_{1}^{2}}\right)=N_{2}\left[p-\frac{\sigma^{2}\left(n_{1}\right)+\left(\bar{n}_{1}\right)^{2}}{N_{1}^{2}}\right], \\
\sigma_{1}^{2}\left(n_{2}\right) & =N_{2}\left[p-\frac{N_{1} p(1-p)+N_{1}^{2} p^{2}}{N_{1}^{2}}\right], \\
\sigma_{1}^{2}\left(n_{2}\right) & =N_{2} p(1-p)\left(1-\frac{1}{N_{1}}\right) .
\end{aligned}
$$

Using Eqs. (7) and (11) in Eq.(5) yields

$$
\sigma^{2}\left(n_{2}\right)=p(1-p) \frac{N_{2}}{N_{1}}\left[N_{1}+N_{2}-1\right] \text {. }
$$


Three special cases can easily be checked against independent calculations, i.e., (1) if $N_{l}=1$, then $\sigma^{2}\left(\mathrm{n}_{2}\right)=N_{2}^{2} p(1-p)$; (2) if $N_{1}>>N_{2}$ and $N_{1}>>1$, then $\sigma^{2}\left(n_{2}\right)=N_{2} p(1-p)$, and (3) if $N_{2}=1$, then $\sigma^{2}\left(n_{2}\right)=p(1-p)$. It will be seen from Eq. (12) that the effect of random source-resampling just once can be substantial. Thus, if $N_{1}=N_{2}=N \gg>1$ then $\sigma^{2}\left(n_{2}\right) \sim 2 N p(1-p)$, so that the variance is doubled just by this one stage. This is true regardless of the number of histories per generation, provided that number is much greater than one.

It is now relatively simple to get a recursion relation for the variances in successive generations of random source resampling. Generalizing Eq. (6) we find that

$$
\sigma_{2, j+1}^{2}\left(n_{2}\right)=\left(N_{j+1} / N_{j}\right)^{2} \sigma_{j+1}^{2}\left(n_{1}\right) .
$$

But $\sigma_{j+1}^{2}\left(n_{1}\right)=\sigma_{j}^{2}\left(n_{2}\right)$, so that

$$
\sigma_{2, j+1}^{2}\left(n_{2}\right)=\left(N_{j+1} / N_{j}\right)^{2} \sigma_{j}^{2}\left(n_{2}\right) .
$$

From Eq. (9),

$$
\sigma_{1, j+1}^{2}\left(n_{2}\right)=N_{j+1}\left[p(1-p)-\sigma_{j}^{2}\left(n_{2}\right) / N_{j}^{2}\right]
$$

Using Eqs.(13) and (14) in the generalization of Eq.(5) yields

$$
\sigma_{j+1}^{2}=N_{j+1} p(1-p)+\sigma_{j}^{2} \frac{N_{j+1}}{N_{j}^{2}}\left(N_{j+1}-1\right),
$$

where $\sigma_{\mathrm{j}} \equiv \sigma_{\mathrm{j}}\left(\mathrm{n}_{2}\right)$.

If all the $N_{j}^{\prime}$ 's are equal and equal to $N_{l}$, and if $N_{l}>>1$

$$
\begin{aligned}
& \sigma_{j+1}^{2} \approx \sigma_{j}^{2}+N p(1-p), \\
& \sigma_{j}^{2} \approx(j+1) N p(1-p),
\end{aligned}
$$

i.e., the variance grows linearly with $\mathrm{j}$.

The fact that the variance grows linearly with $\mathrm{j}$ is not surprising. Some such growth is to be expected for infinite media, just on the basis of arguments in Ref. 5. Here, in Ref. 5 terminology, fluctuations are injected in each generation, unopposed by any restoring force. But the injected variance is rather large. In fact if, as in the one-group JAERI problem, $p=1 / 2$, then after 500 generations the variance in the last-generation absorption rate will be equal to $500 \mathrm{x}$ $1000=5 \times 10^{5}$, so that the standard deviation has grown to 707 in an absorption rate of 1000 . It seems desirable to use a less noisy population control method for problem configurations with very low leakage. One notable weakness of random source-resampling is that it injects large variance even if, as in some of our test problems, the number of fission sites is always equal to the required number of source sites. 


\section{Appendix B}

\section{Theory of Variance Induced Through Use of Collision Sites As Potential Fission Sites}

Consider again a one-group two-core configuration with infinitely large cores. Potential fission sites are now to be taken at collision sites. Suppose that $N_{s}$ source neutrons are injected into the whole system, with $p_{1}$ the probability that a starter will go into core 1 . We want to compute the variances induced in regionwise source distributions just by the process of selection of new source sites, again neglecting the motion of neutrons. Let $p_{a}$ be the probability that a colliding neutron will be absorbed, with $p_{s} \equiv 1-p_{a}$. Further, let $n_{1}$ be the number of source neutrons entering core $1, n_{c}$ the number of collisions these neutrons undergo, and $n_{2}$ the final number of first-generation fission sites in core 1 . We use here the same probability notation as in Appendix A. By definition

$$
\begin{aligned}
\sigma^{2}\left(n_{2}\right) & =\sum_{n_{2}}\left(n_{2}-\bar{n}_{2}\right)^{2} P_{2}\left(n_{2}\right) \\
& =\sum_{n_{1}} \sum_{n_{2}} \sum_{n_{c}}\left[n_{2}-\left(\bar{n}_{2} \mid n_{c}\right)+\left(\bar{n}_{2} \mid n_{c}\right)-\bar{n}_{2}\right]^{2} P_{c}\left(n_{2} \mid n_{c}\right) P_{c 1}\left(n_{c} \mid n_{1}\right) P_{1}\left(n_{1}\right),
\end{aligned}
$$

But one can show, as in Appendix A, that

$\sum_{n_{1}} \sum_{n_{2}} \sum_{n_{c}}\left[n_{2}-\left(\bar{n}_{2} \mid n_{c}\right)\right]\left[\left(\bar{n}_{2} \mid n_{c}\right)-\bar{n}_{2}\right] P_{2 c}\left(n_{2} \mid n_{c}\right) P_{c 1}\left(n_{c} \mid n_{1}\right) P\left(n_{1}\right)=0$

Therefore

$\sigma^{2}\left(n_{2}\right)=\sigma_{1}^{2}\left(n_{2}\right)+\sigma_{2}^{2}\left(n_{2}\right)$

where, after summation over $\mathrm{n}_{2}$,

$$
\begin{aligned}
& \sigma_{1}^{2}\left(n_{2}\right)=\sum_{n_{1}} \sum_{n_{c}} \sigma^{2}\left(n_{2} \mid n_{c}\right) P_{c 1}\left(n_{c} \mid n_{1}\right) P_{1}\left(n_{1}\right), \\
& \sigma_{2}^{2}\left(n_{2}\right)=\sum_{n_{1}} \sum_{n_{c}}\left[\left(\bar{n}_{2} \mid n_{c}\right)-\bar{n}_{2}\right]^{2} P_{c 1}\left(n_{c} \mid n_{1}\right) P_{1}\left(n_{1}\right) .
\end{aligned}
$$

Now the weight of each collision site is equal to $\bar{v} \Sigma_{f} / \Sigma_{t}$, and the mean number of offspring at each site is taken to be $\bar{v} \Sigma_{f}\left(k \Sigma_{t}\right)$. Normally $k 1$, so that $\bar{v} \Sigma_{f}\left(k \Sigma_{t}\right)<1$. Fission sites will then be selected from among collision sites by roulette. In the notation above $\boldsymbol{\sigma}^{2}\left(n_{2} \mid n_{c}\right)$ is the variance in the number of roulette survivors, given the number of collisions. Suppose that, in our model problem, both cores are identical and just critical, i.e. $\bar{v} \Sigma_{f} / \Sigma_{a} \equiv k_{o}=1$, and that $k=k_{o}$. Then $\bar{v} \Sigma_{f} /\left(k \Sigma_{t}\right)$ is equal to $\Sigma_{a} / \Sigma_{t}=p_{a}$. The probability of surviving roulette is then equal to $p_{a}$, and $\boldsymbol{\sigma}^{2}\left(n_{2} \mid n_{c}\right)=n_{c} p_{a}\left(1-p_{a}\right)$. Thus 
$\boldsymbol{\sigma}_{1}^{2}\left(n_{2}\right)=\bar{n}_{c} p_{a}\left(1-p_{a}\right)=N_{s} p_{1}\left(1-p_{a}\right)$

Now we need to compute $\boldsymbol{\sigma}_{2}^{2}\left(n_{2}\right)$. To do this we use the fact that

$\left(\bar{n}_{2} \mid n_{c}\right)=p_{a} n_{c}, \bar{n}_{2}=n_{1}=p_{a} \bar{n}_{c}$,

so that

$$
\begin{aligned}
& \sigma_{2}^{2}\left(n_{2}\right)=p_{a}^{2} \sum_{n_{c}} \sum_{n_{1}}\left[n_{c}-\left(\bar{n}_{c} \mid n_{1}\right)+\left(\bar{n}_{c} \mid n_{1}\right)-\bar{n}_{c}\right]^{2} P_{c 1}\left(n_{c} \mid n_{1}\right) P_{1}\left(n_{1}\right), \\
& \sigma_{2}^{2}\left(n_{2}\right)=p_{a}^{2}\left\{\sum_{n_{c}} \sum_{n_{1}}\left[n_{c}-\left(\bar{n}_{c} \mid n_{1}\right)\right]^{2} P_{c 1}\left(n_{c} \mid n_{1}\right) P_{1}\left(n_{1}\right)+\sum_{n_{1}}\left[\left(\bar{n}_{c} \mid n_{1}\right)-\bar{n}_{c}\right]^{2} P_{1}\left(n_{1}\right)\right\}, \\
& \sigma_{2}^{2}\left(n_{2}\right)=p_{a}^{2}\left[\sum_{n_{1}} \sigma^{2}\left(n_{c} \mid n_{1}\right) P_{1}\left(n_{1}\right)+\sigma^{2}\left(n_{1}\right) / p_{a}^{2}\right] .
\end{aligned}
$$

One can show that $\sigma^{2}\left(n_{c} \mid n_{1}\right)=n_{1} p_{s} / p_{a}^{2}$, so that

$$
\sum_{n_{1}} \sigma^{2}\left(n_{c} \mid n_{1}\right) P_{1}\left(n_{1}\right)=N_{s} p_{1} p_{s} / p_{a}^{2}
$$

while $\boldsymbol{\sigma}^{2}\left(n_{1}\right)=N_{s} p_{1}\left(1-p_{1}\right)$. Thus

$$
\sigma^{2}\left(n_{2}\right)=N_{s} p_{1}\left[2\left(1-p_{a}\right)+\left(1-p_{1}\right)\right]
$$

It will be convenient, at this point, to change our notation slightly. Below we designate the cores as "core A" and "core B". Further, $\mathrm{n}_{1}^{\mathrm{A}}$ and $n_{1}^{B}$ will now be the numbers of firstgeneration starters in each core. The variances $\sigma^{2}\left(n_{1}^{A}\right)$ and $\sigma^{2}\left(n_{1}^{B}\right)$ are both equal to $\mathrm{N}_{\mathrm{s}} / 4$. If we take potential source sites at absorption sites then, following the logic of Appendix A, $\mathrm{n}_{2}^{\mathrm{A}}=\mathrm{n}_{1}^{\mathrm{A}}, \mathrm{n}_{2}^{\mathrm{B}}=\mathrm{n}_{1}^{\mathrm{B}}$, i.e. the number of fission sites in each core is equal to the number of initial source sites in that same core. Thus $\sigma^{2}\left(n_{2}^{A}\right)=\sigma^{2}\left(n_{2}^{B}\right)=N_{s} / 4$. Define $m_{1}^{A}=n_{2}^{A}, \mathrm{~m}_{1}^{\mathrm{B}}=\mathrm{n}_{2}^{\mathrm{B}}$, $M=m_{1}^{A}+m_{1}^{B}$, so that $m_{1}^{A}$ and $\mathrm{m}_{1}^{\mathrm{B}}$ are the numbers of neutrons entering the random sourceresampling process. Similarly $\mathrm{m}_{2}^{\mathrm{A}}$ and $\mathrm{m}_{2}^{\mathrm{B}}$ are the numbers of neutrons exiting the sourceresampling process, and $m_{2}^{A}+m_{2}^{B}=N_{s}$.

Under these conditions we see from Appendix A that

$$
\begin{aligned}
\sigma^{2}\left(m_{2}^{A}\right) & =\sigma^{2}\left(m_{2}^{B}\right)=2 N_{s} p_{1}\left(1-p_{1}\right) \\
& =N_{s} / 2
\end{aligned}
$$


since $p_{1}=1 / 2$. As in Appendix A the computed variances are variances in the numbers of secondgeneration source neutrons entering each core.

Suppose we now take the collision sites as potential source sites, so that now $\mathrm{M}$ will fluctuate. We wish to compute $\sigma^{2}\left(m_{2}^{A}\right)$. To do this we rewrite, in appropriately modified notation, Eqs. (3) and (5) of Appendix A.

$$
\begin{aligned}
& \sigma^{2}\left(m_{2}^{A}\right)=\sigma_{1}^{2}\left(m_{2}^{A}\right)+\sigma_{2}^{2}\left(m_{2}^{A}\right), \\
& \sigma_{1}^{2}\left(m_{2}^{A}\right)=\sum_{m_{1}^{A}} \sum_{m_{1}^{B}} \sigma^{2}\left(m_{2}^{A} \mid m_{1}^{A}, m_{1}^{B}\right) P\left(m_{1}^{A}, m_{1}^{B}\right), \\
& \sigma_{2}^{2}\left(m_{2}^{A}\right)=\sum_{m_{1}^{A}} \sum_{m_{1}^{B}} \sigma^{2}\left(\overline{m_{2}^{A}} \mid m_{1}^{A}, m_{1}^{B}\right) P\left(m_{1}^{A}, m_{1}^{B}\right),
\end{aligned}
$$

where $P\left(m_{1}^{A}, m_{1}^{B}\right)$ is the joint probability distribution of $m_{1}^{A}$ and $m_{1}^{B}$, and

$$
\left(\bar{m}_{2}^{A} \mid m_{1}^{A}, m_{1}^{B}\right)=N_{s} m_{1}^{A} / M
$$

We compute $\sigma_{1}^{2}\left(m_{2}^{A}\right)$ first, writing

$$
\begin{aligned}
& \sigma_{1}^{2}\left(m_{2}^{A} \mid m_{1}^{A}, m_{1}^{B}\right)=N_{s} p(1-p), \quad p \equiv m_{1}^{A} / M, \\
& \sigma_{1}^{2}\left(m_{2}^{A}\right)=N_{s} \sum_{m_{1}^{A}} \sum_{m_{2}^{A}} \frac{m_{1}^{A}}{M}\left(1-\frac{m_{1}^{A}}{M}\right) P\left(m_{1}^{A}, m_{2}^{A}\right) .
\end{aligned}
$$

Here and below we write $\bar{m}=M / 2, m_{1}^{A}=\bar{m}+\delta^{A}, m_{1}^{B}=\bar{m}+\delta^{B}$, and assume that $\delta^{4}, \delta^{B}<<\bar{m}$. To second order in the $\delta$ 's, with $\delta^{T} \equiv \delta^{A}+\delta^{B}$,

$$
\begin{aligned}
r^{A} & \equiv m_{1}^{A} / M=\left(\bar{m}+\delta^{A}\right) /\left(2 \bar{m}+\delta^{T}\right) \\
& =\frac{1}{2}\left[1+\left(\delta^{A} / \bar{m}\right)\right]\left[1-\left(\delta^{T} / 2 \bar{m}\right)+\left(\delta^{T} / 2 \bar{m}\right)^{2}\right], \\
r^{A} & =\frac{1}{2}\left[1+\left(\delta^{A} / \bar{m}\right)-\left(\delta^{T} / 2 \bar{m}\right)-\left(\delta^{A} \delta^{T} / 2 \bar{m}^{2}\right)+\left(\delta^{T} / 2 \bar{m}\right)^{2}\right], \\
& =\frac{1}{2}\left\{1+(1 / 2 \bar{m})\left(\delta^{A}-\delta^{B}\right)-\left(1 / 4 \bar{m}^{2}\right)\left[\left(\delta^{A}\right)^{2}-\left(\delta^{B}\right)^{2}\right]\right\},
\end{aligned}
$$

and to second order in $1 / N_{\mathrm{s}}$ 
$\bar{r}^{A}=\frac{1}{2}$

Further, again to second order,

$\overline{\left(r^{A}\right)^{2}}=\frac{1}{4}\left\{1+\overline{\left(\delta^{A}-\delta^{B}\right)^{2}} /(2 \bar{m})^{2}-\left[\overline{\left(\delta^{A}\right)^{2}-\left(\delta^{B}\right)^{2}}\right] /\left(4 \bar{m}^{2}\right),\right\}$

so that, to leading order in $1 / N_{\mathrm{s}}$

$\sigma_{1}^{2}\left(m_{2}^{A}\right)=N_{s} / 4$

Turning to $\sigma_{2}^{2}\left(m_{2}^{A}\right)$ we see that

$$
\begin{aligned}
\sigma_{2}^{2}\left(m_{2}^{A}\right) & =N_{s}^{2} \sigma^{2}\left(r^{A}\right) \\
& =N_{s}^{2}\left[\overline{\left(r^{A}\right)^{2}}-\left(\overline{r^{A}}\right)^{2}\right] \\
\sigma_{2}^{2}\left(m_{2}^{A}\right) & =\left(N_{s}^{2} / 16 \bar{m}^{2}\right)\left[2 \sigma^{2}\left(m_{1}^{A}\right)-\overline{2 \delta^{A} \delta^{B}}\right] .
\end{aligned}
$$

To evaluate $\overline{\delta^{A} \delta^{B}}$ we proceed as follows. Rewrite

$\delta^{A}=m_{1}^{A}-\bar{m}=n_{2}^{A}-n_{1}^{A}+n_{1}^{A}-\bar{m}$,

$\delta^{B}=n_{2}^{B}-n_{1}^{B}+n_{1}^{B}-\bar{m}$,

noting that $n_{1}^{A}=\left(\overline{n_{2}^{A}} \mid n_{1}^{A}\right), n_{1}^{B}=\left(\overline{n_{2}^{B}} \mid n_{1}^{B}\right)$. Intuitively it seems clear that

$$
\begin{aligned}
\overline{\left(n_{2}^{A}-n_{1}^{A}\right)\left(n_{2}^{B}-n_{1}^{B}\right)} & =\overline{\left(n_{2}^{A, B}-n_{1}^{A, B}\right)\left(n_{1}^{B, A}-\bar{m}\right)} \\
& =0,
\end{aligned}
$$

since, for example, fluctuations in $n_{2}^{A}$ about $n_{1}^{A}$ are due to scattering-roulette in core A, a process independent of all others in the system. Thus

$$
\begin{aligned}
\overline{\delta^{A} \delta^{B}}=\overline{\left(n_{1}^{A}-\bar{m}\right)\left(n_{1}^{B}-\bar{m}\right)} & =-\sigma^{2}\left(n_{1}^{A}\right) \\
& =-N_{s} / 4 .
\end{aligned}
$$


We see that

$$
\begin{aligned}
& \sigma^{2}\left(m_{2}^{A}\right)=\frac{1}{4}\left[2 \sigma^{2}\left(m_{1}^{A}\right)+\frac{1}{2} N_{s}\right]+N_{s} / 4 \\
& \sigma^{2}\left(m_{2}^{A}\right)=\frac{1}{4}\left\{2 N_{s} p_{1}\left[2\left(1-p_{a}\right)+\frac{1}{2}\right]+\frac{1}{2} N_{s}\right\}+N_{s} / 4, \\
& \sigma^{2}\left(m_{2}^{A}\right)=\left(N_{s} / 2\right)\left(1-p_{a}\right)+N_{s} / 2 .
\end{aligned}
$$

Thus in this case, the random source-resampling tends to cut in half the variance due to the scattering-roulette process. Still if $p_{a}<<1$ the "excess" variance introduced by this process is about equal to the variance introduced by random source-resampling.

In Eq. (21) the quantity $\sigma^{2}\left(m_{2}^{A}\right)$ is the variance of the fission source constructed in the course of the first generation. As in Appendix A one can easily generalize this expression to one which gives the variance, $\sigma_{j}^{2}$, in the $\mathrm{j}$ 'th generation, and one finds that

$$
\sigma_{j}^{2}=\left(N_{s} / 2\right)\left[j\left(1-p_{a}\right)+(j+1) / 2\right]
$$

On comparing Eq. (22) with Eq. (16) of Appendix A, we see that the variance induced by the use of collision sites is equal to $\left(N_{s} / 2\right)\left(1-p_{a}\right)$. From Eq. (8) of this appendix we find that the corresponding variance just before invoking random source-resampling is equal to $N_{s}\left(1-p_{a}\right)$. Apparently the random source-resampling smears over both cores the variance due to the use of collision sites, cutting this variance in half. It seems likely that, if random source-resampling is replaced by a more innocuous population control process, the variance due directly to population control will decrease, but that due to scattering-roulette will increase, tending towards the value $N_{s}\left(1-p_{a}\right)$. 


\section{Appendix C}

\section{Theory of Variances in Averages Over Generations}

In Appendices A and B we computed variances in numbers of starters, in a given region, for each generation. These variances do not tell us directly what will be the variance in the average-over-generations of these numbers. To compute such variances one must take into account the correlations between fluctuations in different generations. We consider such correlations in the same test problems as treated in the earlier Appendices. Here we use the ideas of Ref. 2, and make only a slight change in the Ref. 2 notation; it will be convenient to rewrite Eq. (11) of Ref. 2 in the form

$\underline{\psi}^{(n+1)}=H \underline{\psi}^{(n)} / k^{(n)}+\underline{\varepsilon}^{(n+1)}$,

where $\underline{\varepsilon}^{(n+1)}$ is the statistical error in the MC computation of $\underline{\psi}^{(n+1)}$ given $\underline{\psi}^{(n)}$. We note that here, and in Appendix D, the approach is general, not depending on the details of the Monte Carlo method. From Eq. (1), retaining only linear terms in the $\delta$ 's, we see that, again from Ref. 2 ,

$\underline{\delta}^{(n+1)}=A \delta^{(n)}+\underline{\varepsilon}^{(n+1)}, \quad n=0,1, \ldots, N$,

$\underline{\varepsilon}^{(o)} \equiv \underline{\delta}^{(o)}$, where $\underline{\delta}^{(o)}$ is the error in the source guess and $N$ is the number of generations.

From (2)

$\underline{\boldsymbol{\delta}}^{(n)}=\sum_{m=0}^{n} A^{n-m} \underline{\boldsymbol{\varepsilon}}^{(m)}$

In the present test problems there are only 2 unknowns, i.e., the integrated sources in regions 1 and 2 . If the system is critical and the cores are decoupled, then $\mathrm{H}=\mathrm{I}$, and

$\underline{U}_{o} \underline{\tilde{\tau}}^{T}=\frac{1}{2}\left(\begin{array}{l}1 \\ 1\end{array}\right)\left(\begin{array}{l}1 \\ 1\end{array}\right)^{T}=\frac{1}{2}\left(\begin{array}{ll}1 & 1 \\ 1 & 1\end{array}\right)$,

so that

$A=\frac{1}{2}\left(\begin{array}{cc}1 & -1 \\ -1 & 1\end{array}\right)$.

We then have $A^{n}=A$ for $n>0$, so that

$\underline{\boldsymbol{\delta}}^{(n)}=A \sum_{m=0}^{n-1} \underline{\boldsymbol{\varepsilon}}^{(m)}+\underline{\boldsymbol{\varepsilon}}^{(n)}$ 
From (6)

$E\left\{\left[\underline{\boldsymbol{\delta}}^{(n)}\right]^{2}\right\}=\sum_{m=o}^{n-1} E\left\{\left[\underline{\varepsilon}^{(m)}\right]^{T} A^{2} \underline{\boldsymbol{\varepsilon}}^{(m)}\right\}+\sigma^{2}\left[\underline{\mathcal{\varepsilon}}^{(n)}\right]=n \gamma^{2}+\sigma^{2}$,

where

$$
\sigma^{2} \equiv E\left\{\left[\underline{\varepsilon}^{(n)}\right]^{2}\right\} \text { and } \gamma^{2} \equiv E\left\{\left[\underline{\varepsilon}^{(m)}\right]^{T} A^{2} \underline{\varepsilon}^{(m)}\right\} .
$$

Thus if in the recursion relation for the $\delta$ 's we retain only linear terms, then the variance in the n'th generation fission source will increase linearly in $\mathrm{n}$, a result which is now independent of the details of the Monte Carlo method.

If $\underline{\boldsymbol{\delta}}_{A V}^{(N)} \equiv \frac{1}{N} \sum_{m=1}^{N} \underline{\boldsymbol{\delta}}^{(m)}$, then

$\underline{\delta}_{A V}^{(N)}=\frac{1}{N}\left\{\sum_{m=1}^{N}\left[m A \underline{\varepsilon}^{(N-m)}+\varepsilon^{(m)}\right]\right\}$.

From Eq. (7) it is easy to show that

$E\left\{\left[\underline{\boldsymbol{\delta}}_{A V}^{(N)}\right]^{2}\right\} \rightarrow \frac{\gamma^{2}}{3} N$ as $N \rightarrow \infty$

since

$$
\sum_{m=1}^{N} m^{2} \rightarrow \frac{1}{3} N^{3}
$$

Thus not only the variances in the fission sources of individual generations, but also the variance in the average-over-generations goes to infinity as the number of generations goes to infinity. 


\section{Appendix D}

\section{Theory of Coupling and Source Distribution Fluctuations}

Finally in this Appendix we consider the effect of point-to-point coupling in the twopoint model of Appendix C. Whereas, for small fluctuations, the arguments of the earlier Appendices are exact for the given one-group problem, this is no longer true here. First, as soon as the cores are coupled the fission source shapes are relevant and the eigenvalue equation is no longer a $2 \times 2$ matrix equation. Secondly, if each of the two slabs is infinite the probability that an average neutron will be transferred from one to the other is vanishingly small. Still for us the $2 \times 2$ matrix model still retains some interest for at least one reason. We think it interesting to ask whether, in this simple model, any threshold coupling level is required if the variance in source shape is to remain finite. Suppose that, in this model,

$$
H=\left(\begin{array}{cc}
1-a, & a \\
a, & 1-a
\end{array}\right) .
$$

Now

$$
\begin{aligned}
& A=\left(\begin{array}{ll}
\frac{1}{2}-a, & a-\frac{1}{2} \\
a-\frac{1}{2}, & \frac{1}{2}-a
\end{array}\right)=\frac{(1-2 a)}{2}\left(\begin{array}{cc}
1, & -1 \\
-1, & 1
\end{array}\right), \\
& A^{n}=c^{n} \tilde{A}, \tilde{A} \equiv \frac{1}{2}\left(\begin{array}{cc}
1, & -1 \\
-1, & 1
\end{array}\right), c \equiv(1-2 a), n>0 .
\end{aligned}
$$

Arguing as in Appendix C one finds that, in place of Eq. (7) of this earlier Appendix, we now have

$\underline{\delta}_{A V}^{(n)}=\frac{1}{N}\left\{\sum_{m=1}^{N}\left[S_{m} \tilde{A} \underline{\boldsymbol{\varepsilon}}^{(N-m)}+\underline{\boldsymbol{\varepsilon}}^{(m)}\right]\right\}$,

where $S_{m}=\sum_{\ell=1}^{m} c^{\ell}<(1-c)^{-1}$.

From (4) it will be seen that for large N,

$$
\sigma^{2}\left[\underline{\delta}_{A V}^{(N)}\right] \rightarrow \mathrm{L} \leq \frac{1}{N^{2}}\left[N \gamma^{2} /(1-c)^{2}\right]
$$

with $\gamma^{2}$ defined as in Appendix C. Clearly, then, within the limits of our approximations 
$\sigma^{2}\left[\underline{\delta}_{A V}^{(N)}\right] \rightarrow 0$ as $N \rightarrow \infty$

Unfortunately, our argument does not tell us what happens for any finite number of histories per generation. 
Distribution for ANL-01/15 Internal:

D. J. Hill, RAE, 208

H. S. Khalil, RAE, 208

E. K. Fujita, RAE, 208

D. Weber, RAE, 208 R. Hill, RAE, 208

J. Binder, RAE, 208

J. Roglans, RAE, 208

C. Grandy, RAE, 208

R. Blomquist, RAE, 208

G. Palmiotti, RAE, 208

C. Stenberg, RAE, 208

T. Taiwo, RAE, 208

K. Grimm, RAE, 208

J. Stillman, RAE, 208

R. Hwang, RAE, 208

R. Lell, RAE, 208

J. Morman, RAE, 208

C. Adams, RAE, 208

B. Toppel, RAE, 208

R. McKnight, RAE, 208

J. Cahalan, RAE, 208

K. Derstine, RAE, 208

T. Wei, RAE, 208

R. Hwang, RAE, 208

E. Gelbard, RAE, 208

T. Fanning, RAE, 208

R. W. Schaefer, NT-AW

L. W. Deitrich, OTD-ER, 208

J. Helt, OTD-ER, 208

Y. I. Chang, OTD-ER, 208

S. K. Battacharyya, TD, 360

P. Finck, TD, 360

J. Deen, TD, 360

N. Hanan, TD, 360

Y. Gohar, TD, 360

J. Carpenter, PNS, 360

External: ANL-East Library

ANL-West Library

RAE Division (30)

A. Forster, LANL

R. Little, LANL R. MacFarlane, LANL

M. Chadwick, LANL

Rick Anderson, LANL

Tom McLaughlin, LANL

M. Westfall, ORNL 
C. Parks, ORNL

M. DeHart, ORNL

Lester Petrie, ORNL

Hamilton Hunter, ORNL

Luiz Leal, ORNL

Tim Valentine, ORNL

Ian Gould, ORNL

B. Kirk, ORNL

David Heinrichs, LLNL

Song Huang, LLNL

Rich Evarts, LLNL

Blair Briggs, INEEL

Nigel Smith, Serco Assurance-Winfrith

Adolf Garcia, DOE

Hoyt Johnson, DOE-EM-21

James Felty, DOE-DP

Kim Loll, DOE

John Evans, DNFSB

Jerry McKamy, DOE-EH

Matthew Hutmaker, DOE-DP

Ray Schwartz, DOE

Richard Stark, DOE-EH

William Lake, DOE-RW

Ivon Fergus, DOE

Burt Rothleder, DOE-EH

Enrico Sartori, OECD/NEA

Ali Nouri, OECD/NEA

Tom Reilly, SRS-EH

C. Dunford, BNL (NNDC)

P. Oblizinsky, BNL (NNDC)

Robert Wilson, DOE

Todd Taylor, BWXT-Idaho 